\title{
Evaluation and treatment of peroneal neuropathy
}

\author{
Jennifer Baima $\cdot$ Lisa Krivickas
}

Published online: 11 March 2008

(C) Humana Press 2008

\begin{abstract}
Peroneal nerve compromise results in the clinical complaint of weakness of the ankle dorsiflexors and evertors. This peripheral origin of foot drop has been reported due to numerous traumatic and insidious causes. Traumatic causes of nerve injury occur in association with musculoskeletal injury or with isolated nerve traction, compression, or laceration. Insidious causes include mass lesions and metabolic syndromes. The peroneal nerve is most commonly interrupted at the knee. However, the sciatic and peroneal nerves may be compromised at the hip and ankle as well. This article reviews the anatomical origin of the nerve, the etiologies of possible nerve damage, evaluation of the patient with peroneal nerve injury, and treatment of this disorder.
\end{abstract}

Keywords Peroneal nerve - Ankle dorsiflexors .

Foot drop $\cdot$ Sciatic nerve injury

\author{
Anatomy \\ The sacral plexus is formed from the L4-S4 ventral rami. \\ The largest nerve in the sacral plexus and in the human body \\ ventral rami and exits the sacral plexus by passing through \\ innervate the short head of the biceps femoris muscle. The \\ J. Baima $(\varangle) \cdot$ L. Krivickas \\ Brigham and Women's Hospital, Boston, MA, USA \\ e-mail: jbaima1@partners.org \\ J. Baima · L. Krivickas \\ Spaulding Rehabilitation Hospital, Boston, MA, USA
} is the sciatic nerve. It receives contributions from the L4-S3 the greater sciatic foramen and traveling inferior to the piriformis muscle. The posterior divisions of the ventral rami ultimately form the common peroneal nerve and anterior divisions of the ventral rami innervate the other three hamstring muscles and ultimately form the tibial nerve. The sciatic nerve divides into the common peroneal (fibular) and tibial nerves proximal to the popliteal fossa. Of note, the same fibers that run laterally in the sciatic nerve to innervate the short head of the biceps femoris are the fibers that will form the common peroneal nerve at the knee. Figure 1 demonstrates the course of the common peroneal nerve, which is also referred to as the common fibular nerve.

The common peroneal nerve then travels across the lateral head of the gastrocnemius muscle. This nerve provides cutaneous innervation to the lateral aspect of the leg below the knee via the lateral cutaneous nerve of the calf. Just distal to the head of the fibula, the nerve becomes subcutaneous. It continues between the peroneus longus muscle and the fibula. At this point, it divides into two main branches, the deep peroneal and superficial peroneal nerves.

The deep peroneal nerve innervates the anterior muscles of the leg by traveling deep to the peroneus longus. This nerve supplies the tibialis anterior, extensor digitorum longus, peroneus tertius, and extensor hallucis longus. These muscles control foot dorsiflexion and toe extension. The tibialis anterior is the strongest contributor to foot dorsiflexion. The extensor digitorum longus and peroneus tertius assist with dorsiflexion. The deep peroneal nerve receives segmental innervation from the L4 through S1 nerve roots.

In the foot, the deep peroneal nerve remains in close proximity to the interosseus membrane with the anterior tibial artery. The nerve supplies the short toe extensors, the extensor digitorum brevis, and extensor hallucis brevis. Also, it provides innervation to the intertarsal joints. Then, it terminates in a cutaneous branch. This branch innervates the web space between the first and second toes [1].

The superficial peroneal nerve travels between the peroneus longus and brevis, the primary ankle evertors, and 
Fig. 1 Distribution of the peroneal nerve [1]. Reprinted with permission from W.B. Saunders

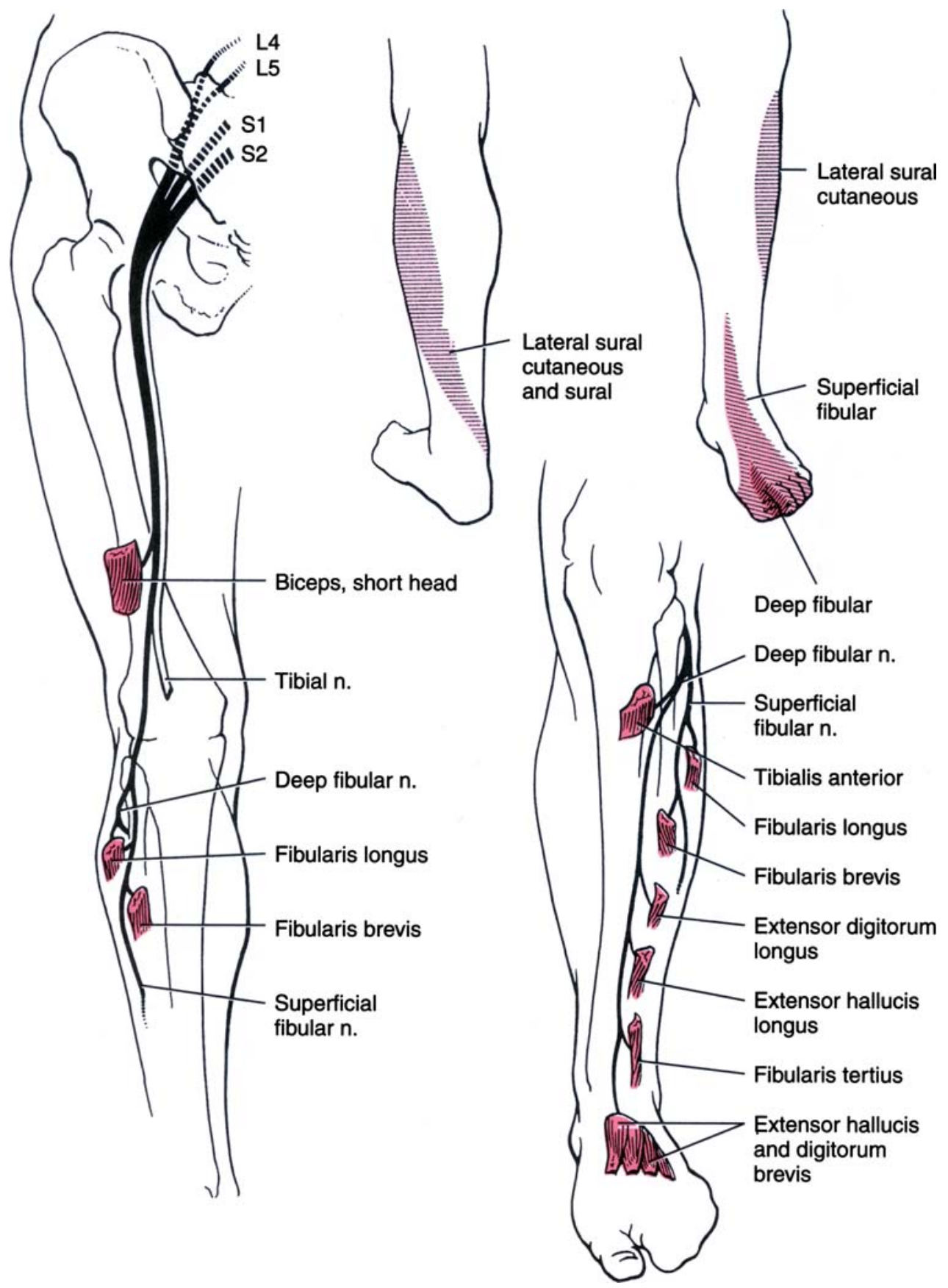

innervates both muscles. The peroneus tertius and extensor digitorum longus also contribute to ankle eversion. Segmental innervation is the same as for the deep peroneal nerve. Near to the midpoint of the lateral lower leg, the nerve becomes subcutaneous. It supplies sensation to the skin of the lateral leg and the dorsum of the foot and toes, sparing the small area between the first two toes and a variable lateral part of the foot [1]. Fifteen to twenty-eight percent of patients have an accessory peroneal nerve that branches off the superficial peroneal to supply the extensor digitorum brevis (usually innervated by the deep peroneal) [2-4].

\section{Etiology}

Peroneal nerve compromise has been reported due to numerous traumatic and insidious causes. Traumatic causes of nerve injury occur in association with musculoskeletal injury or with isolated nerve traction, compression, or laceration. Insidious causes include mass lesions and metabolic syndromes. The common peroneal nerve may be tethered by the tendinous origin of the peroneus longus as it winds around the fibular head and passes through the peroneal tunnel to divide over the fibular neck [5]. Thus, it 
is more susceptible to injury at the knee. However, the sciatic and peroneal nerves may be compromised at the hip and ankle as well.

At the hip, the lateral fibers of the sciatic nerve are the most susceptible to injury. These are the fibers that will form the common peroneal nerve at the knee. The lateral location of the nerve fibers, tethering at the fibular head, and the larger size of the funiculi are likely to be responsible for this susceptibility [6]. Acetabular fracture, femur fracture, or operative repair of these fractures jeopardizes these nerve fibers. Incidence reports range from $16 \%$ to $33 \%$. The most commonly reported complication of hip osteotomy is sciatic nerve paresis. This injury has also been reported with posterior hip dislocation without fracture [7].

Non-operative treatment of hip fractures can result in sciatic injury. This can be avoided by careful monitoring of patients during femoral traction. Given the high complication rate of lower extremity traction, it is no longer commonly performed as definitive treatment. However, this does illustrate that the lateral fibers of the sciatic nerve may be more susceptible to traction injuries at the hip or the knee. Although less frequent, knee dislocation can result in sciatic nerve injury when the nerve is stretched around the posterior femoral condyle [7].

The common peroneal nerve may be injured at the knee with a fracture of the tibia or fibula, especially at the proximal fibula. There is an approximately $1 \%$ incidence of peroneal nerve injury with tibial plateau fracture. Also, this can occur with knee arthrodesis and is a rare complication of total knee arthroplasty, realignment of the knee extensor mechanism, and arthroscopic meniscal repair. Stretch injury of the peroneal nerve can occur after treatment of knee flexion contracture. Utilizing a posterolateral incision and placing a retractor to protect the nerve from traction can help to prevent common peroneal nerve injury during arthroscopic knee surgery [7].

Peroneal neuropathy at the knee has been reported with ligamentous knee injury. In a series of 31 lower extremity sports injuries, 17 were peroneal nerve injuries. Eight of these traumatic injuries were associated with ligamentous injury of the knee. Most of these involved anterior cruciate ligament rupture. This often occurred in conjunction with injury to the lateral, collateral, or posterior cruciate ligament. Of note, three of the peroneal neuropathies in this series were associated with ankle injuries [8].

Ligamentous or bony injury of the ankle may cause peroneal neuropathy. However, nerve damage results more often from treatment of ankle fracture than from the fracture itself. Treatment of ankle injuries with a below-knee splint or cast can damage the peroneal nerve by pressure over the fibular head. Associated lower extremity edema can worsen this complication. This can be avoided by padding the cast or splint in the area of the fibular head and avoiding prolonged pressure on the lateral knee (as can occur during bed rest with lateral rotation of the leg). To follow the patient clinically, the inferior trim lines of the cast can be moved proximally toward the ankle to allow for visualization of active toe extension as documentation of deep peroneal nerve function. In the ankle, iatrogenic injury to the superficial peroneal nerve may occur in surgical incisions placed at the anterior border of the fibula to approach the distal fibula or the anterior tibiofibular joint [7]. Ankle sprain is a common cause of morbidity in the general population, and the ankle is the most commonly injured joint complex among athletes [9]. The mechanism of injury in ankle sprain involves inversion of a plantar flexed ankle. This position applies traction to the peroneal nerve at the fibular head due to stretching of the peroneal muscles [5]. Peroneal nerve injury after ankle sprain was first described by Hyslop in 1941 in a case series of three patients. The mechanism of injury was proposed as a traction injury of the nerve in the posterolateral knee from a sudden force with the patient's foot in plantarflexion and inversion. Although the site of injury is at the fibular head, the symptomatic area is the lateral foot [10]. Concurrent ankle sprain and peroneal neuropathy at the fibular head may be uncommon, but easily misdiagnosed. Patients with ankle sprain often experience lateral ankle pain and eversion weakness from the primary ligamentous injury.

If there has been an injury to the peroneal nerve at the knee during lateral ankle sprain, this could be detected within 2 weeks after injury on electromyographic examination. A series of 66 patients with ankle sprain underwent this evaluation. Eighty-six percent of patients with grade III sprains and seventeen percent of patients with grade II sprains had electrodiagnostic evidence of peroneal nerve injury on needle examination. Nerve conduction studies were normal in most patients with ankle sprain. Three of the patients (10\%) with grade II injury had abnormal sensory studies, and one of the patients with grade III ankle sprain had abnormal motor studies [11].

Of note, over $30 \%$ of the patients with grade III ankle sprain had tenderness of the popliteal fossa on clinical exam at 2-week follow-up. This feature was not present at initial evaluation and occurred only in those patients with electromyographic abnormalities. At 3-month follow-up, electrical abnormalities were only present in two patients. These abnormalities were consistent with reinnervation. Only one patient had transient foot drop, and this did not last past 3 weeks post-injury. Further study in this area could help elucidate the clinical significance of a mild and reversible peroneal neuropathy concurrent with ankle sprain [11].

Iatrogenic injury of the peroneal nerve can occur from direct manipulation during orthopedic surgery as previously mentioned or with prolonged compression during lateral hip and leg rotation with knee flexion as occurs in 
operative positioning. This position is often used in gynecologic as well as abdominal surgery. In addition to positioning during surgery, patients who require prolonged bed rest after surgery may be at risk for peroneal neuropathy. Gravity, the shape of the lower extremity, and muscular imbalance contribute to the tendency of the lower limb to rest in external rotation of the hip and knee flexion while lying supine. This position, together with the bony prominence of the fibular head, may jeopardize the peroneal nerve unless a preventative program is instituted. This involves the use of bilateral padded ankle foot orthoses which maintain the feet in dorsiflexion and the lower limbs in neutral (without external rotation) while the patient is resting in bed. Frequent repositioning of the supine patient can also minimize pressure on the lateral knee.

In addition to peroneal neuropathy, prolonged bed rest is a risk factor for deep venous thrombosis. Currently, the medical community has an increased awareness of the post-operative risk of thromboembolic disease. Pneumatic compression devices may be used in the prevention of this life-threatening disorder. Bilateral peroneal nerve palsies following intermittent pneumatic compression have been reported [12].

Unfortunately, malnutrition is a common comorbid condition with bed rest or prolonged surgery. Since malnutrition leads to weight loss, this may be a complicating factor when considering positioning as the cause of peroneal neuropathy. Since both weight loss and bed rest may result in a neuropathy of subacute onset, chronology does not help to identify the causative factor. Patients who have weight loss, independent of bed rest or compressive lesion, have developed peroneal neuropathy [13]. Bilateral peroneal neuropathy has been reported after bariatric surgery [14]. As more patients undergo this weight loss surgery, we may come to a better understanding of the role of weight loss in peroneal mononeuropathy.

Currently, there is no agreement as to the quantity of weight loss that will result in peroneal nerve damage. The proposed mechanism is loss of fat previously protecting the peroneal nerve. In a series of 116 patients with common peroneal neuropathy, Katirji and Wilbourn describe twenty-five patients with gradual or indeterminate onset of the disorder who had weight loss of more than twenty pounds over 2-3 months [15]. Of interest, peroneal neuropathy has also been reported with prolonged squatting [16]. Biomechanical comparisons of compression of the peroneal nerve in squatting have not been evaluated in terms of body mass index.

Less commonly, peroneal nerve injury occurs as a result of laceration. In a series of 318 common peroneal nerve lesions for which surgery was performed, 39 (12\%) lacerations were identified. These were due to injury from broken glass, knives, boat propellers, chain saws, or lawn mower blades. Three of the 39 patients had continuity of the nerve despite this mechanism of injury and subsequently had better recovery. In this same series, 12 patients had peroneal nerve injuries due to gunshot wound. The majority of these patients still had lesions in continuity [17].

The case series of Kline and colleagues also addressed mass lesions. Forty patients (13\%) had tumors. The most common of these was intraneural ganglion cyst. Other tumors, in order of decreasing frequency, included schwannoma, neurofibroma, osteochondroma, neurogenic sarcoma, focal hypertrophic neuropathy, desmoid tumor, and glomus tumor [17]. In a much smaller study of nine patients presenting with peroneal nerve palsy who underwent MRI, six patients had ganglion cyst, one had a synovial cyst, one had osteochondroma, and one had an aneurysm [18]. Intraneural ganglion cyst appears to be a frequently occurring mass lesion in peroneal neuropathy. Of note, the most common site of intraneural ganglia within the peripheral nervous system is the common peroneal nerve [19].

Osteoarthritis of the knee may contribute to peroneal neuropathy via mechanisms involving nerve stretch or compression. A case of varus knee deformity due to arthritis resulting in peroneal neuropathy at the level of the knee has been reported. There was no evidence of compressive lesion at the time of operative exploration. Consequently, the lesion was thought to be due to repetitive traction injury from varus malalignment. The patient responded to treatment with total knee arthroplasty with attention to prevention of post-operative varus knee laxity [20]. There was no noted lateral knee osteophyte in this patient. However, an osteophyte causing peroneal nerve palsy has been reported in a younger patient [21].

\section{Patient evaluation}

Initially, patients with peroneal neuropathy complain of lateral lower limb and dorsal foot pain. Concurrent low back pain or posterolateral thigh pain suggests L5 radiculopathy. Pain usually precedes sensory changes in a similar distribution. The patient may complain of foot drop as the first manifestation of this disorder. Table 1 presents the various possible lesions resulting in ankle weakness of peripheral neurogenic origin.

The patient's leg should be examined for ecchymosis, edema, or ulcers. Evidence of trauma or vascular comprise may help to determine the cause of the lesion. The peroneal nerve may be palpated as it winds around the head of the fibula. Tapping over this area may reproduce dysesthesia in the lateral calf or foot.

Careful sensory examination can assist with localizing the lesion. The deep peroneal nerve supplies the area between the first and second toes. The remaining dorsum of 
Table 1 Foot drop of peripheral origin

\begin{tabular}{|c|c|c|c|}
\hline Nerve & Motor & Sensory & Weakness \\
\hline L5 nerve root & $\begin{array}{l}\text { Gluteus maximus/medius/minumus, tensor } \\
\text { fasciae latae, semitendinosus, } \\
\text { semimembranosus, biceps femoris, tibialis } \\
\text { posterior, TA, PL, PB, EDB, EHL } \\
\text { (gastrocnemius/soleus) }\end{array}$ & $\begin{array}{l}\text { Lateral leg, lateral calf, } \\
\text { dorsum of foot, and medial } \\
\text { toes }\end{array}$ & $\begin{array}{l}\text { Hip abduction and internal rotation } \\
\text { Knee flexion } \\
\text { Ankle dorsiflexion, inversion, and eversion } \\
\text { Toe extension (plantarflexion) }\end{array}$ \\
\hline $\begin{array}{l}\text { Lumbosacral } \\
\text { plexopathy } \\
\text { a variable } \\
\text { presentation: } \\
\text { example is lesion } \\
\text { proximal to sciatic } \\
\text { nerve }\end{array}$ & $\begin{array}{l}\text { Gluteus medius/ minimus, tensor fascia lata, } \\
\text { semitendinosus, semimembranosus, biceps } \\
\text { femoris, tibialis posterior, flexor digitorum } \\
\text { longus, abductor hallucis, flexor digitorum } \\
\text { brevis, flexor hallucis brevis, abductor } \\
\text { digiti minimi, adductor hallucis, interossei, } \\
\text { TA, EDL, EHL, EDB, EHB, PT, PL, PB, } \\
\text { gastrocnemius, soleus (partial adductor } \\
\text { magnus) }\end{array}$ & $\begin{array}{l}\text { Posterior thigh, lateral calf, } \\
\text { dorsal and plantar foot } \\
\text { (sparing lateral foot) }\end{array}$ & $\begin{array}{l}\text { Hip internal rotation and abduction, knee } \\
\text { flexion, ankle dorsiflexion, } \\
\text { plantarflexion, inversion and eversion } \\
\text { Toe extension (partial hip adduction) }\end{array}$ \\
\hline Sciatic nerve & $\begin{array}{l}\text { Semitendinosus, semimembranosus, biceps } \\
\text { femoris, tibialis posterior, flexor digitorum } \\
\text { longus, abductor hallucis, flexor digitorum } \\
\text { brevis, flexor hallucis brevis, abductor } \\
\text { digiti minimi, adductor hallucis, interossei, } \\
\text { TA, EDL, EHL, EDB, EHB, PT, PL, PB, } \\
\text { gastrocnemius, soleus (partial adductor } \\
\text { magnus) }\end{array}$ & $\begin{array}{l}\text { Lateral calf, dorsal and } \\
\text { plantar foot (sparing } \\
\text { lateral foot) }\end{array}$ & $\begin{array}{l}\text { Knee flexion } \\
\text { Ankle dorsiflexion, plantarflexion, } \\
\text { inversion and eversion } \\
\text { Toe extension (partial hip adduction) }\end{array}$ \\
\hline $\begin{array}{l}\text { Common peroneal } \\
\text { nerve }\end{array}$ & TA, EDL, EHL, EDB, EHB, PT, PL, PB & $\begin{array}{l}\text { Lateral calf and dorsum of } \\
\text { foot (sparing lateral and } \\
\text { plantar foot) }\end{array}$ & $\begin{array}{l}\text { Ankle dorsiflexion and eversion } \\
\text { Toe extension }\end{array}$ \\
\hline Deep peroneal nerve & TA, EDL, EHL, EDB, EHB, PT & $\begin{array}{l}\text { Area between great and } \\
\text { second toes }\end{array}$ & $\begin{array}{l}\text { Ankle dorsiflexion and partial } \\
\text { eversion }>\text { inversion } \\
\text { Toe extension }\end{array}$ \\
\hline $\begin{array}{l}\text { Superficial peroneal } \\
\text { nerve }\end{array}$ & PL, PB & $\begin{array}{l}\text { Lateral calf and dorsum of } \\
\text { foot (sparing lateral foot) }\end{array}$ & Ankle eversion \\
\hline
\end{tabular}

${ }^{\mathrm{a}} \mathrm{TA}=$ tibialis anterior, $\mathrm{PL}=$ peroneus longus, $\mathrm{PB}=$ peroneus brevis, $\mathrm{EDB}=$ extensor digitorum brevis, $\mathrm{EHL}=$ extensor hallucis longus, $\mathrm{EDL}=$ extensor digitorum longus, $\mathrm{PT}=$ peroneus tertius, $\mathrm{EHB}=$ extensor hallucis brevis

the foot is innervated by the superficial peroneal nerve, except for a small area laterally. Sensation of the plantar foot is spared in lesions of the peroneal nerve.

All muscles of the lower limb should be examined for weakness and compared to the contralateral side. Weakness of the ankle dorsiflexors, toe extensors, and ankle evertors, commonly referred to as foot drop, is suggestive of peroneal neuropathy. In a series of 303 patients with foot drop (including that of central neurogenic origin), almost $31 \%$ of these lesions were due to peroneal neuropathy. Another $19.7 \%$ of these cases were due to L5 radiculopathy [22]. Less frequently, lumbosacral plexopathy can result in weakness in a similar distribution.

Often, patients with L5 radiculopathy or sciatic neuropathy will have similar deficits as those with common peroneal neuropathy. The tibialis posterior, innervated by the tibial nerve, receives its main segmental innervation from L5. This muscle provides the majority of ankle inversion. If ankle inversion is weak, the lesion involves more than the common peroneal nerve.
Examination of normal and pathologic reflexes can further narrow the differential. The patellar tendon reflex is innervated by the L2 through L4 nerve roots. The Achilles reflex is primarily from S1. Absent or diminished reflexes suggest a peripheral origin of foot drop. The presence of pathologic reflexes, such as a Babinski reflex, suggest foot drop of central origin. Table 1 below presents the most common peripheral causes of foot drop. Since peroneal neuropathy appears clinically most similar to L5 radiculopathy, L4 and S1 were excluded from this table. As previously mentioned, the $\mathrm{L} 4, \mathrm{~L} 5$, and $\mathrm{S} 1$ roots contribute to the common peroneal nerve.

\section{Imaging and electrodiagnostic testing}

Plain radiographs of the knee and ankle should be obtained to evaluate for concurrent fracture, mass lesion, or arthritis if the history suggests one of these etiologies. Lumbar MRI can provide evidence of L5 radiculopathy if radiographs 
are negative. Knee and ankle MRI can further elucidate a bony lesion or demonstrate intraneural ganglia. In order to view the anatomy of the actual nerve, 3-tesla MRI is necessary [23]. These machines are now becoming available at large centers. Kim and his group [18] suggest that knee MRI should be performed in all cases of non-traumatic peroneal nerve palsy since intraneural ganglia may be the most common etiology. Another newer technique to assess the area around the fibular head is high-resolution sonography. Visser suggests that ultrasonography is an accessible and easy way to evaluate the common peroneal nerve in its superficial location [24].

Electrodiagnostic studies assist with confirming the diagnosis of peroneal neuropathy, excluding alternative diagnoses, and determining prognosis. Recommended examination includes motor nerve conduction studies of the peroneal nerve and tibial nerve and sensory nerve conduction studies of the sural and superficial peroneal nerves. In general, if the lesion involves demyelination, focal slowing or conduction block (amplitude loss at more proximal stimulation sites) can be seen. If the lesion is due to axon loss, compound muscle action potential amplitudes will be decreased at all stimulation sites. Needle electromyographic exam can further localize the lesion. Routine muscles examined for this study include two muscles innervated by the deep peroneal nerve, one muscle innervated by the superficial peroneal nerve, the tibialis posterior, another muscle innervated by the tibial nerve (i.e., medial gastrocnemius), and the short head of the biceps femoris. If any of the muscles supplied by the peroneal nerve are abnormal, further muscles supplied by the L5 nerve root but not the peroneal nerve (i.e., tibialis posterior) should be evaluated to exclude radiculopathy, lumbosacral plexopathy, or sciatic neuropathy [2].

\section{Treatment}

Pain is the earliest symptom in peroneal neuropathy and may be the most difficult to treat. Currently available agents for neuropathic pain include the following: topical lidocaine, capsaicin, selective serotonin reuptake inhibitors, antiepileptics, opioids, and $\mu$-receptor agonists. As these provide symptomatic relief only, the choice of medication depends on comorbidities and possible adverse effects. A review of these choices is beyond the scope of this article, but treatment should be individualized to the patient.

Modalities such as heat and ice can also provide effective pain relief. However, patients with sensory loss should be carefully observed during the use of modalities to prevent skin damage. Also, superficial ice treatment may injure the peroneal nerve at the fibular head when applied incorrectly. This was reported in the case of a football player who applied ice to a hamstring muscle strain [25]. Skin damage from superficial cold application does not usually occur with the use of a protective barrier for the skin and limited duration of therapy. Superficial heat may result in burn injury. This can occur with lying on a heating pad or prolonged therapy.

Iontophoresis has been suggested for the relief of pain in peroneal neuropathy [26]. This involves the transcutaneous delivery of ionic medications (i.e.corticosteroids) into the soft tissue with the use of electrical current. Although there has been little evidence to suggest the actual absorption of steroids into human soft tissue, this may provide symptomatic relief for the patient and is generally well tolerated.

Weakness in peroneal neuropathy may lead to functional gait impairment. Muscle atrophy can become clinically apparent at 2 weeks after injury. If there is transection of a nerve or a complete axon loss lesion, it will be of no use to attempt to strengthen the denervated muscles. If there is compression, it is best to relieve the offending agent prior to a trial of strengthening. If the patient has subtle peroneal nerve injury, strengthening may help with functional recovery. If the patient has complete loss of strength, passive range of motion may be all that is possible. It is imperative to maintain proper ankle range of motion so that residual heel cord contracture will not preclude the ability to walk. Recently, peroneal nerve stimulators have been gaining in popularity for the treatment of foot drop of central etiology $[27,28]$. These devices require an intact functioning peroneal nerve and are not useful in patients with peripheral nerve injury.

Patients with sensory loss should check their feet daily to prevent progression of ulcers. A simple lace-up ankle sleeve with medial and lateral support may assist a patient with proprioceptive loss. In peripheral neuropathy, it is rare to have proprioceptive loss until the late stages of disease. If sciatic or peroneal palsy is severe enough to demonstrate proprioceptive loss, the patient is more likely to have gait impairment and require intervention [29].

If the patient has weakness of the toe extensors only, as would occur in distal deep peroneal neuropathy, sturdy footwear may be all that is needed to optimize gait. A rocker-bottom shoe may decrease the energy required for ambulation. If the patient has isolated superficial peroneal nerve palsy, he may benefit from a shoe insert with a lateral wedge to prevent supination of the foot from weakness of the evertors. If the patient has a proximal deep peroneal neuropathy, he may be unable to dorsiflex the ankle. Ankle dorsiflexion is necessary to clear the toes while ambulating. An ankle foot orthosis maintains the foot in neutral so that the patient can achieve a normal gait pattern. If the lesion is at the level of the common peroneal nerve, the foot may tend toward plantarflexion and inversion. This patient 
would also require an ankle foot orthosis for toe clearance during gait. Patients with common peroneal neuropathy require a more solid, less flexible AFO than those with only deep peroneal neuropathy.

All patients with weakness should stretch daily to prevent contracture. Equinovarus foot deformity is a common complication of ankle dorsiflexion weakness. It is essential that the patient maintain his range of motion to have the ability to ambulate. After contracture has developed, the patient may be unable to tolerate bracing. If bracing is not effective, the tibialis posterior tendon can be transferred to the dorsum of the foot to restore active dorsiflexion. This is often performed in conjunction with fusion of the subtalar joint and after one year post-injury [7]. Removal of the offending agent, lesion, or activity is the best treatment of peroneal neuropathy. Consequently, prompt recognition and diagnosis is imperative in order to preserve maximum function. Initially, treatment may be conservative in most peroneal nerve lesions. Open wounds associated with peroneal nerve palsy should undergo immediate surgical exploration [30]. Other lesions are followed clinically and may be investigated electromyographically. If there is no evidence of functional recovery, surgery may be performed at 3-7 months from injury. Operative technique and time to intervention vary according to the nature of the injury. Neurolysis yields the best outcome. End-to-end suture repair is preferable to graft repair, and shorter grafts yield better outcomes. In a series of 381 patients who underwent operative management for common peroneal neuropathy, antigravity or greater ankle dorsiflexion function was achieved in $75 \%$ of patients with grafts less than $6 \mathrm{~cm}$ in length [17].

\section{References}

1. Jenkins DB. The Leg. In: Hollinshead's functional anatomy of the limbs and back. 8th ed. Philadelphia: WB Saunders; 2002. pp. 327-50.

2. Preston DC, Shapiro BE. Peroneal neuropathy. In: Electromyography and neuromuscular disorders. Philadelphia: Elsevier; 2005. pp. 343-54.

3. Dimitru D. Focal peripheral neuropathies. In: Electrodiagnostic medicine. Philadelphia: Hanley and Belfus; 1995. p. 898.

4. Kimura J. Sacral plexus and its principle nerves. In: Electrodiagnosis in diseases of nerve and muscle. Oxford: Oxford University Press; 2001. pp. 22-5.

5. McCrory P, Bell S, Bradshaw C. Nerve entrapments of the lower leg. Sports Med. 2002;32(6):371-91.

6. Feinberg JH, Nadler SF, Krivickas LS. Peripheral nerve injuries in the athlete. Sports Med. 1997;24(6):385-408.

7. Epps CH. Complications in orthopaedic surgery. Philadelphia: JB Lipincott Company; 1994. pp. 473, 563, 568, 608-10, 757, 846, 1064-65, 1226-27, 1229.

8. Krivickas LS, Wilbourn AJ. Peripheral nerve injuries in athletes: a case series of over 200 injuries. Semin Neurol. 2000;20(2):22532 .
9. Braun B. Effects of ankle sprain in a general clinic population six to 18 months after medical evaluation. Arch Fam Med. 1999;8:143-8.

10. Hyslop G. Injuries to the deep and superficial peroneal nerves complicating ankle sprain. Am J Surg. 1941;11(2):436-8.

11. Nitz AJ, Dobner JJ, Kersey D. Nerve injury and Grades II and II ankle sprains. Am J Sports Med. 1985;13(3):177-82.

12. Fukuda $\mathrm{H}$. Bilateral peroneal nerve palsy caused by intermittent pneumatic compression. Intern Med. 2006;45(2):93-4 Epub 2006 Feb 15.

13. Sotaniemi KA. Slimmer's paralysis-peroneal neuropathy during weight reduction. J Neurol Neurosurg Psychiatry. 1984;47(5):564-6.

14. Elias WJ, Pouratian N, Oskouian RJ, Schirmer B, Burns T. Peroneal neuropathy following successful bariatric surgery. J Neurosurg. 2006;105:631-5.

15. Katirji MB, Wilbourn AJ. Common peroneal mononeuropathy: a clinical and electrophysiologic study of 116 lesions. Neurology. 1988;38:1723-8.

16. Togrol E. Bilateral peroneal nerve palsy induced by prolonged squatting. Mil Med. 2000;165(3):240-2.

17. Kim DH, Murovic JA, Teil RL, Kline DG. Management and outcomes in 318 operative common peroneal nerve lesions at the LSU Health Sciences Center. Neurosurgery. 2004;54:1421-9.

18. Kim JY, Ihn YK, Kim JS, Chun KA, Sung MS, Cho KH. Nontraumatic peroneal nerve palsy: MRI findings. Clin Radiol. 2007;62:58-64.

19. Spinner RJ, Atkinson JL, Tiel RL. Peroneal intraneural ganglia: the importance of the articular branch. A unifying theory. J Neurosurg. 2003;99:330-43.

20. Fetzer GB, Prather H, Gelberman RH, Clohisy JC. Progressive peroneal nerve palsy in a varus athritic knee. JBJS. 2004;86A(7):1538-40.

21. Flores LP, Koerbel A, Tatagiba M. Peroneal nerve compression resulting from fibular head osteophyte-like lesions. Surg Neurol. 2005;64(3):249-52.

22. Van Langenhove M, Pollefliet A, Vanderstraeten G. A retrospective electrodiagnostic evaluation of footdrop in 303 patients. Electromyogr Clin Neurophysiol. 1989;29:145-52.

23. Spinner RJ, Atkinson JL, Scheithauer BW, Rock MG, Birch R, Kim TA, Kliot M, Kline DG, Tiel RL. Peroneal intraneural ganglia: the importance of the articular branch. Clinical series. J Neurosurg. 2003;99(2):319-27.

24. Visser LH. High-resolution sonography of the common peroneal nerve: detection of intraneural ganglia. Neurology. 2006;67:1473-5.

25. Moeller JL, Munroe J, McKeag DB. Cryotherapy induced common peroneal nerve palsy. Clin J Sports Med. 1997;7:212-6.

26. Edwards PH, Wright ML, Hartman JF. A practical approach for the differential diagnosis of chronic leg pain in the athlete. Am J Sports Med. 2005;33(8):1241-9.

27. Weber DJ, Stein RB, Chan KM, Loeb GE, Richmond FJ, Rolf R, James K, Chong SL. BIONic WalkAide for correcting foot drop. Conf Proc IEEE Eng Med Biol Soc. 2004;6:4189-92.

28. Shefller LR, Hennessey MT, Naples GG, Chae J. Peroneal nerve stimulation versus an ankle foot orthosis for correction of footdrop in stroke: impact on functional ambulation. Neurorehabil Neural Repair. 2006;20(30):355-60.

29. Hagiwara Y, Hatori M, Kokubun S, Miyasaka Y. Gait characteristics of sciatic nerve palsy-a report of four cases. Ups J Med Sci. 2003;108:221-7.

30. Garazzo D, Ferraresi S, Buffatti P. Surgical treatment of common peroneal nerve injuries: indications and results: a series of 62 cases. J Neurosurg Sci. 2004;48(3):105-12. 